$\begin{array}{ll}\text { le portiQue } & \text { Le Portique } \\ \text { Revue de philosophie et de sciences humaines }\end{array}$

$20 \mid 2007$

Gilles Deleuze et Félix Guattari : Territoires et devenirs

\title{
Révolution et Hybridité : Le transcorps
}

\section{Bernard Andrieu}

\section{OpenEdition}

\section{Journals}

\section{Édition électronique}

URL : http://journals.openedition.org/leportique/1360

DOI : $10.4000 /$ leportique. 1360

ISSN : $1777-5280$

\section{Éditeur}

Association "Les Amis du Portique"

Édition imprimée

Date de publication : 15 décembre 2007

ISSN : 1283-8594

\section{Référence électronique}

Bernard Andrieu, « Révolution et Hybridité : Le transcorps », Le Portique [En ligne], 20 | 2007, mis en ligne le 06 novembre 2009, consulté le 26 mars 2021. URL : http://journals.openedition.org/leportique/ 1360 ; DOI : https://doi.org/10.4000/leportique.1360

Ce document a été généré automatiquement le 26 mars 2021.

Tous droits réservés 


\title{
Révolution et Hybridité : Le transcorps
}

\author{
Bernard Andrieu
}

\section{Le trans}

1 Renverser le capital ne suffira pas. La libération ne peut pas s'achever dans une inversion des sexes, genres, des classes sociales. Le retournement des usagers contre l'école, l'asile, l'usine, s'il participe du processus de régulation socio-économique programmée par le capital, conduit à l'épuisement faute d'une "société libérée de l'exploitation $»^{0}$.

2 De même qu'il y a une transversalité dans la psychanalyse, il y a une transexualité pour dépasser l'homosexualité et ce qui serait sa perversion, sa transgression: «il s'agit donc en fait moins d'homosexualité que trans-sexualité : il s'agit de définir ce que serait la sexualité dans une société libérée de l'exploitations capitaliste et des rapports d'assujettissement qu'elle développe à tous les niveaux de l'organisation sociale $»^{0}$.

3 La réflexion sur le transcorps est contemporaine de celle sur le genre engagée par Irigaray, Wittig et Oakley en offrant la possibilité de déconstruire la masculinisation du corps sans parvenir à se détacher de la survalorisation du féminin. Le transcorps suppose $^{0}$ l'affirmation d'identification de genre par une révolution hormonales, symboliques et orgasmique.

4 Le rhizome fait le multiple plus qu'il ne l'énonce. L'échec d'une biologie qui ne serait pas moléculaire, l'ouvrage de Felix Guattari est publié en 1977 sous le titre de la Révolution moléculaire, pourrait trouver dans la botanique les principes d'un rhizomorphisme : à la fois connexions, hétérogénéités, multiplicités et asignifiances, le rhizome va au-delà des regénérations, des reproductions des hydres et des méduses. "Les systèmes arborescents sont des systèmes hiérarchiques qui comportent des centres de signifiance et de subjectivation" (Rhizome, 1976, p. 47-48). La totalité organique n'existe pas, seules les combinaisons, les permutations et les utilisations transforment la matière subjectivante sans parvenir à l'épuiser. 
Dans le rhizome l'intactibilité est le principe de la détéritorialisation et de la de stratification (Rhizome, 1976, p.9) car le contact entre les lignes de fuite est inattribuable même si la vitesse crée un événement haptique qui affecte le corps sans organes. Il n'y a pas d'organe du toucher aucune peau-surface dont l'intensité laisserait une trace. Il n'y a plus de différence entre le touchant et le touché, entre le corps qui ressent et la constitution multisensorielle de ce corps. Les multiplicités rhizomatiques ne produiront pas une arborescence du dedans-dehors, de la profondeur et de la surface car « la ligne de fuite fait partie du rhizome » (Rhizome, 1976, p. 28).

6 Ainsi la guêpe ne touche pas l'orchidée, mais il y a à la fois un devenir-guêpe de l'orchidée et devenir-orchidée de la guêpe. Le toucher rhizomatique opère dans l'hétérogène sans reprise homogène dans une sensation définie. Antigénéalogique, le rhizome haptique ne trouve ni dans l'objet tangible ni dans le sujet tactile une origine ou une finalité. Toucher est une carte du tendre plutôt qu'un calque arborescent et il n'est pas réductible. Le devenir mouvement du rhizome haptique est l'inverse de la culture arborescente du tactile: celle-ci fait de la peau la surface d'une profondeur archéologique du soi, le tactile restant les racines du tangible.

\section{Contre les micro-cristallisations}

7 Ce qu'il faut combattre c'est ce qui interdit, empêche, censure et limite le mouvement du vivant dans l'être au nom de normes, d'impératifs qui relèvent d'une conception homogène du corps sociale et du corps du sujet : tout me porte à penser, en effet, que les nouvelles micro-cristallisations fascistes qui ne cessent de proliférer sous nos yeux, au sein du pouvoir d'État, au sein des partis, des syndicats, des groupuscules, à travers l'information, les attitudes racistes... $»^{0}$.

8 Ce n'est pas l'homme qui pense, qui contrôle la pulsion; il faut consentir à l'abandon de la maîtrise de soi et de l'autre pour livrer le désir en passant du chaos au cerveau. Le cerveau n'est pas à traiter comme l'objet constitué des neurosciences afin de servir de cautions au neurocomportementalisme et aux dépistages des aneuraux et des déneuraux, nouvelles catégories de la pathologie neurocognitive du développement.

9 Car le cerveau devient sujet, il est l'esprit même, ou plutôt « superjet », que « le concept devient l'objet comme créé $»^{0}$.

\section{Devenir hybride}

10 Plutôt que de subir son environnement, le sujet doit, selon Marie-Hélène Bourcier, « ... s'inventer, se créer, prendre soin de soi, développer un style de vie, une technologie de soi, une éthique de soi $»^{0}$. Il ne s'agit pas de rester toujours le même puisque l'être est en devenir, multiple, variable et interactif. L'hybride est justement ce qui fait advenir l'être dans l'existant : «Est-ce que l'on doit sans arrêt se déplacer, être en mouvement continuel, ne jamais être là où on vous attend $»^{0}$. Toujours en mouvement, en déplacement physique et mental, l'individu mondialisé doit modifier sans cesse ses représentations en intégrant les informations. Cette "immanence plurielle $»^{0}$ implique une réalisation du soi à travers les actes corporels de plus en plus hybridés par les moyens de communication comme le satellite, l'internet, le portable, l'informatique et 
par les modes d'amélioration de l'existence que sont le viagra, les hormones, les antivirus, les cultures in vitro...

Gilles Deleuze et Felix Guattari précisent combien être et devenir hybride n'ont pas la même signification. Être hybride est statique, limitant les possibilités de transformations et de mutations: "la constitution de ces hybrides ne nous fait pourtant pas davantage avancer dans le sens d'un véritable devenir... on ne rompt pas avec le schéma d'arborescence, on n'atteint pas au devenir ni au moléculaire $»^{0}$. Être hybride c'est le devenir, ne pas être dans l'être mais dans la multiplicité possible dont l'incarnation sera provisoire et éphémère. L'hybride n'est pas stable, il varie sans cesse en modifiant son intensité et ses états. La vitesse est remplacée aujourd'hui par le mouvement, forçant à une mobilité mentale, corporelle et sexuelle ce qui favorise l'isolement pour ceux et celles qui ne peuvent s'hybrider aux changements de l'environnement.

12 Cette multiplicité ne se contrôle pas, elle déborde les frontières identitaires en hybridant le sujet à ses autres possibilités d'être. Il devient un autre en lui-même, à la manière d'une performance accomplie sans s'en rendre compte, parce que l'interaction environnementale actualise ce qu'il pourrait être, mais dont la possibilité n'avait pas été jusque-là créée par l'interaction avec l'environnement. L'essente de l'être corporel est à déconstruire pour y découvrir, non pas sa permanence et son homogénéité, mais de nouveaux modes d'existence. L'hybridation sert de techniques pour vivre ces existences en se connectant à des postures de genres, à des objets interactifs et à des actions. L'hybridation déstabilise l'être installé dans une stase identitaire: en restant toujours le même, l'être peut considérer l'hybride comme un intrus, un danger ou un virus.

13 À la déterritorialisation dans l'espace correspond le devenir hybride dans le temps, la capacité « du système corps-tête à devenir " $^{0}$. La prise de possession du dedans du corps révèle la multidimensionalité du corps : car la possession, l'implantation, la greffe, la chimérisation, la transgenèse, l'hybridation sont autant de procédés biotechnoculturels qui utilisent la plasticité du vivant. Si Spinoza ignorait ce dont le corps est capable, la révolution moléculaire de Guattari est la conséquence du corps sans organe, de son débordement.

Car l'organicité du corps a pu faire croire en la limitation ontologique, sinon métaphysique, de la morphogenèse. Le débordement de la forme est le principe à mettre en œuvre par des « reconversions, d'Agencements qui débordent de toutes parts le corps, le Moi, l'individu $»^{0}$. Le CsO est " un ensemble de pratiques ${ }^{0}$ qui déconstruit, dépose, perd, détruit l'organisation de l'organicité même: le corps hypocondriaque, corps paranoïque, corps schizo, corps drogué, corps masochiste. Les yeux sont-ils faits pour voir, les poumons pour respirer, la bouche pour avaler, le cerveau pour penser? Dès la découverte des gènes hox et la fabrication par Nicole le Douarin des chimères caille-poulet, le jeu du génie génétique a prouvé le déterminisme développemental malgré le changement de territoire.

\section{Décorporer l'organisme}

Le passage du déterminisme développemental aux techniques transgéniques a accompli la déterritorialisation ontogénétique par la création non seulement de Cso mais d'organes sans corps : cœur, foie, sans, cellules, ovocytes... Si le génie génétique n'était 
pas mis en relation avec les genders studies, comme le réalise justement Bordo, la modification des «zones d'intensité » ne serait pas entièrement dénaturalisée et desorganisée : Le champ d'immanence ou plan de consistance doit être construit: or il peut l'être dans des formations sociales très différentes, et par des agencements très différents, pervers, autistes, scientifiques, mystiques, politiques qui n'ont pas le même type de corps sans organes. Il sera construit morceau par morceau, lieux, conditions, techniques ne se laissant pas réduire les uns aux autres ${ }^{\circ}$.

En distinguant l'organisme et le corps, la déconstruction morphogénétique du corps comme forme définie et fonctionnelle devient une strate parmi d'autre du CoS, comme " un phénomène d'accumulation, de coagulation, de sédimentation qui lui impose des formes, des fonctions, des liaisons, des organisations dominantes, hiérarchisées, des transcendances organisées pour en extraire un travail utile $»^{0}$. "S'il faut beaucoup de strates ", c'est pour se dégager de l'organisme, de la signifiance et de la subjectivation comme autant d'obstacles épistémologiques et épistémiques qui contiennent l'organe dans le corps. La déterritorialisation des gènes par la transgenèse et des sexes rejoint le projet queer.

La déstratification évite la cristallisation corporelle qui identifie et réduit le vivant à la normativité du biopouvoir sans dépasser les limites du vivable. Ce dépassement passe par une critique des représentations, des modèles, des objets produits sous l'évidence de l'objectivité. Ce caractère idéologique des strates vient introduire la possibilité des pratiques intensificatoire en maintenant le corps après l'œuf, en découvrant l'œuf comme « milieu d'intensité pure $"^{0}$ sans image du corps établie ou à retrouver.

La différence intensive du désir rend les formes contingentes et un plan d'immanence en évitant les effets cristallisant de la transcendance biopolitique. Ainsi, même dans le $\mathrm{CsO}$, il faut "distinguer ce qui est composable ou non sur le plan $»^{0}$. Car les décompositions du génome humain et du genre ont commencé de révéler les connexions discontinues et les liaisons transversales dans toutes les possibilités objectives du vivant au delà des formes normatives de la division sexuelle, du développement eugénique ou encore de la création OGM.

\section{Produire le moléculaire en nous}

19 Le devenir est le moyen de l'hybridation en déchaînant les chaînes Homme/femme, Genes Hox/Hom, espaces/Individu, Nature/culture : « devenir femme, devenir enfant, devenir-animal, végétal ou minéral, devenir moléculaire de toute sorte, devenir particules $»^{0}$. La révolution moléculaire doit « commencer par la fin $»^{0}$. Il ne s'agit plus de devenir quelqu'un ou quelque chose, s'identifier mais d'être le plus proche « de ce qui est en train de devenir ». Ainsi « tous les devenirs sont déjà moléculaires »: la zone moléculaire et l'espace corpusculaire définissent une hybridation potentielle par les effets de bordure, de composition et d'implantation. Il faut aller à l'inverse du clonage, "non pas imiter le chien mais composer son organisme avec autre choses, de telle manière qu'on fasse sortir de l'ensemble ainsi composé, des particules qui seront canines en fonction du rapport de mouvement et de repos ou du voisinage moléculaire dans lequel elle entend $»^{0}$. des particules» en "produisant en nous-même une femme moléculaire, créer une 
femme moléculaire $»^{0}$. Plutôt que d'imiter, le devenir hybride passe à travers et en dessous les affrontements molaires et autres machines duelles. Si « le corps qu'on nous vole pour fabriquer des organismes opposables $\|^{0}$, alors l'assignation au sexe, à l'identité, à la sexualité, au travail, à la classe nous prive de "l'anorganisme du corps ", du genre et du queer. "L'émission de particules $»^{0}$ est le modèle de transcorps à la fois pour décorporer le vivant et pour définir une microdynamique de la matière biologique: l'idéalisation paradigmatique du devenir femme qui serait «la clef des autres devenirs ${ }^{0}$ participe encore d'un ordre biologique de l'hybridation assez conforme à la déconstruction de la sexuation, de la reproduction, de l'utérus artificiel, de la FIV et la sélection génétique eugénique.

\section{Conclusion}

En allant vers «un devenir imperceptible» comme "fin immanente du devenir $»^{0}$, l'ensemble des composants moléculaires du cosmos rendra «imperceptif, indiscernables, impersonnel $»^{0}$, l'hybridation. La question de jusqu'où défaire le genre, posée par Judith Butler, est celle des limites de la désubjectivation forçant chacun désormais à être hors de soi : "L'institution de nouveaux modes de réalité passe notamment par la corporalisation (embodiement), pour laquelle le corps n'est pas compris comme un fait établi et statique, mais comme un processus de maturation, un devenir qui, en devenant autre, excède la norme, la retravaille et nous montre que les réalités auxquels nous pensions être confinés ne sont pas gravées dans le marbre $»^{0}$.

\section{NOTES}

0. F. GUATTARI, «Trois milliards de pervers à la barre ", La Révolution moléculaire, 10/18, 1973, p. 331.

o. Ibid.

0. Stéphanie NICOT, Alexandra AUGUST-MERELLE, Changer de sexe, Identités transexuelles, Éd. Cavalier Bleu, 2006.

0. F. GUATTARI, Micro-politique du fascisme, 1974.

0. G. DELeUze et F. GUATTARI, «Du chaos au cerveau », Qu'est-ce que la philosophie ?, Paris, Éd. de Minuit, 1991.

0. M.-H. BOURCIER, «Savoirs-pouvoirs sont partout. Comment résister?", Zoo n 1, Q comme Queer, 1997, p. 76-83, ici p. 80.

0. C. DESCHAMPS, «Just be queer? ", Zoo n 1, Q comme Queer, 1997, p. 110-111, ici p. 110.

0. G. GENETTE, L'Eeuvre de l'art, Paris, Seuil, 1997

0. G. Deleuze et F. GUATTARI, Mille Plateaux, Paris, Minuit, p. 389.

0. Ibid., p. 216.

0. F. GUATTARI, Les Trois Écologies, Paris, Galilée, 1989, p. 58.

0. Ibid., p. 186.

0. Ibid., p. 195

0. Ibid., p. 197. 

0. Ibid., p. 202.
0. Ibid., p. 204.
0. Ibid., p. 333.
o. Ibid., p. 334.
0. Ibid., p. 334 et 340 .
o. Ibid., p. 335-336.
0. Ibid., p. 337.
0. Ibid., p. 338.
0. Ibid., p. 341.
0. Ibid., p. 340.
0. Ibid., p. 342.
0. Ibid., p. 343.
0. J. BUTLER, « Hors de soi », Défaire le genre, Paris, Éd. Amsterdam, 2006 [2002], p. 43.

\section{RÉSUMÉS}

Le rhizome fait le multiple plus qu'il ne l'énonce. L'échec d'une biologie qui ne serait pas moléculaire, l'ouvrage de Felix Guattari est publié en 1977 sous le titre de la Révolution moléculaire, pourrait trouver dans la botanique les principes d'un rhizomorphisme : à la fois connexions, hétérogénéités, multiplicités et asignifiances, le rhizome va au-delà des regénérations, des reproductions des hydres et des méduses.

The transbody: revolution and hybridism This article offers a reflection on the transbody contemporary to that on gender engaged by Irigaraz, Wittig and Oakley. The transbody brings the possibility to deconstruct masculinisation of the body, without succeeding in taking some distance with the overvaluation of the feminine. Finally, the transbody presupposes a statement of gender identification through a hormonal, symbolic and orgasmic revolution.

Revolution und Hybridität : der Transkörper Der „Rhizome“ ist eher Vermehrfachung als Formulierung. Das Werk von Felix Guattari erscheint 1977 unter dem Titel „Révolution moléculaire“ (Molekularische Revolution). Es könnte in der Botanik die Prinzipien Rhizomorphismus gefunden haben.

\section{AUTEUR}

\section{BERNARD ANDRIEU}

Bernard Andrieu est PR en Épistémologie du corps et des pratiques corporelles à la Faculté du sport de Nancy Université. Dernier ouvrage paru et dirigé : Le Dictionnaire du corps en SHS, Éd. du CNRS, 2006. 